\title{
Approche inverse améliorée pour la minimisation du retour élastique de pièces embouties
}

\author{
Slim Ben-Elechi* — Hakim Naceur** \\ Catherine Knopf-Lenoir** — Jean-Louis Batoz*** \\ * University of Birmingham, Edgbaston, Birmingham B15 2TT United Kingdom \\ ** Laboratoire Roberval, Université de Technologie de Compiègne, FRE 2833 \\ CNRS BP 20529 - F-60205 Compiègne cedex \\ *** Institut Supérieur d'Ingénierie de la Conception (GIP-InSIC) \\ 27 rue d'Hellieule, F-88100 St-Diés Des Vosges \\ s.benelechi@bham.ac.uk
}

\begin{abstract}
RÉSUMÉ. Dans ce travail on s'intéresse à la minimisation de la déformation élastique des pièces embouties suite au retrait des outils en fin d'emboutissage. Nous utilisons une méthode de surface de réponse (MSR) basée sur l'approximation diffuse (AD) et un algorithme spécifique pour la recherche du minimum. Pour la simulation d'emboutissage, nous adoptons l'approche inverse avec une amélioration des contraintes pour prendre en compte les effets de cambrage et de décambrage dans la zone de brin libre. Les efforts internes et la forme finale de la pièce emboutie sont utilisés pour le calcul du retour élastique en adoptant une formulation lagrangienne actualisée. Deux tests d'emboutissage sont utilisés pour la validation de la procédure d'optimisation proposée en référence aux codes commerciaux Abaqus $^{\circledR}$ et Stampack ${ }^{\circledR}$.

ABSTRACT. This work is focused on the minimization of the elastic deformed drawn parts after tools removing. We use a Response Surface Method based on Diffuse Approximation and a specific algorithm for the optimum searching. For the deep drawing process simulation, we use the Inverse Approach with stress improvements to take into account the bending and unbending effects. Internal efforts with the final workpiece shape are used for the springback calculation, using an Updated Lagrangian Formulation. Two deep drawing benchmarks are studied to validate the proposed procedure with reference to Abaqus ${ }^{\circledR}$ and Stampack ${ }^{\circledR}$ commercial codes.

MOTS-CLÉS: surface de réponse, emboutissage, retour élastique, approche inverse, optimisation.
\end{abstract}

KEYWORDS: response surface, deep drawing, springback, inverse approach, optimization.

DOI:10.3166/REMN.17.349-372 @ 2008 Lavoisier, Paris 


\section{Introduction}

Le retour élastique est la déformation élastique qui se produit en fin d'emboutissage après enlèvement des outils. Cette déformation change la géométrie de la tôle emboutie, elle peut ainsi causer des difficultés lors d'assemblage. Une bonne prédiction du retour élastique est d'une grande importance pour la conception des outils dans l'industrie automobile, aéronautique... (Gelin et al., 1999; KenHechiro, 2001). Habituellement pour éliminer le retour élastique on procède à des séries de corrections de la forme d'outils ce qui demande beaucoup de temps et devient coûteux.

L'optimisation de paramètres du procédé d'emboutissage (Barlet et al., 1996; Naceur et al., 1999; Guo et al., 2000 ; Delamézière et al., 2002) mène souvent à de nombreuses évaluations de la fonction coût. En général la connaissance des gradients, nécessaire pour les algorithmes de minimisation n'est pas toujours disponible ni accessible, particulièrement quand on utilise des logiciels commerciaux qui sont souvent fermés (boîte noire). La méthode de surface de réponse est employée (Roux et al., 1998 ; Stander, 2001 ; Myers et al., 2002 ; Zhang et al., 2002) pour remplacer le modèle d'analyse complexe et coûteux par une approximation basée sur des résultats calculés en quelques points dans l'espace de conception. La MSR peut être utilisée pour diminuer le coût d'évaluation de fonctions.

La MSR a été bien établie pour des problèmes de physique (Myers et al., 2002), cependant ses applications à la simulation des modèles mécaniques reste encore un domaine exploratoire. Roux et al., (1998) ont présenté des techniques de conception expérimentales et les équations de régression pour l'optimisation de structures. Un algorithme génétique est employé par Kurtaran et al., (2002) pour choisir l'ensemble optimal d'expériences entre $\left\langle\mathrm{r}^{\mathrm{n}}\right.$ factoriel $»$ conceptions. Une application à la simulation de l'emboutissage des tôles minces par la méthode dynamique explicite (Ls-Dyna) et la MSR est présentée par Stander (2001). La MSR combinée avec la méthode des éléments finis stochastiques sont employées par Kleiber et al., (2002) pour l'évaluation de fiabilité pour les transformations de métaux. Une méthode hybride originale combinant la recherche de modèle avec la MSR est proposée par Zhang et al. (2002). Ils ont employé une base de fonctions radiales pour représenter la surface de réponse.

Dans cet article, nous rappelons dans un premier temps la formulation classique de l'approche inverse (AI) (Guo et al., 1990 ; Batoz et al., 1996; Naceur et al., 2002) pour la simulation rapide d'emboutissage, puis nous détaillons une nouvelle amélioration de l'AI avec introduction de moments de cambrage-décambrage pour améliorer la distribution des contraintes en fin d'emboutissage. La troisième section présente le problème général d'optimisation. Dans la quatrième section on présente la formulation du modèle de surface de réponse utilisé, suivie de la conception des plans d'expérience et des stratégies pour construire des modèles de régression (cinquième section) et de l'algorithme d'optimisation (sixième section). La septième section présente les applications numériques. Les résultats obtenus sont ensuite discutés, ils montrent la rapidité et la robustesse de la méthodologie proposée. 


\section{Simulation numérique d'emboutissage}

De nos jours beaucoup de logiciels commerciaux (Ls-Dyna, Pam-Stamp, Stampack...) proposent de plus en plus de modules pour la simulation implicite ou/et explicite de retour élastique. Toutefois le temps de calcul fastidieux et la sensibilité des résultats de retour élastique à la densité de maillage et à la taille de pas de temps (Yen 1996 ; Rojek et al., 1998 ; Guo et al., 2001 ; Stander et al., 2001 ; Bui et al., 2003) rendent ces modules difficiles à employer, en particulier si on souhaite optimiser la géométrie des outils pour minimiser les effets de retour élastique.

\subsection{Brève description de l'approche inverse}

Dans l'approche inverse, la prédiction de la forme initiale du flan à partir de la forme finale de la pièce connue est faite en un seul pas de calcul en comparant directement les configurations initiale et finale (Guo et al., 1990 ; Batoz et al., 1998 ; Naceur et al., 2002).

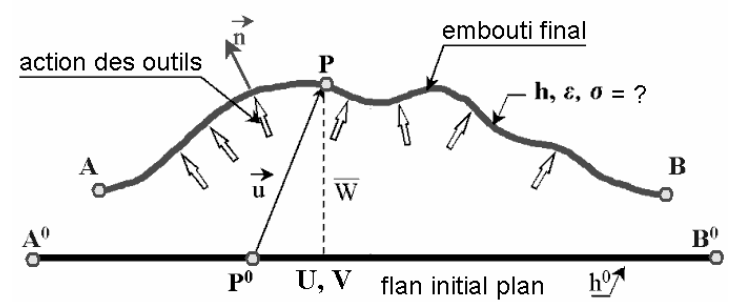

Figure 1. Description de l'approche inverse

Dans l'AI, le principe des travaux virtuels est établi sur l'embouti final connu C :

$$
W=\sum_{e}\left(\int_{V^{e}} \boldsymbol{\varepsilon}^{*^{T}} \boldsymbol{\sigma} d V-\int_{V^{e}} \mathbf{u}^{* T} \mathbf{f} d V\right)=0
$$

où $\boldsymbol{\varepsilon}^{*}$ et $\mathbf{u}^{*}$ sont les déformations et les déplacements virtuels, $\boldsymbol{\sigma}$ les contraintes de Cauchy et $\mathbf{f}$ les forces externes. Les vecteurs position d'un point matériel q dans ses positions initiale et finale, peuvent être exprimés par :

$$
\begin{aligned}
& \mathbf{x}_{q}^{0}=\mathbf{x}_{p}^{0}+z^{0} \mathbf{n}^{0}=\mathbf{x}_{p}-\mathbf{u}_{p}+z^{0} \mathbf{n}^{0} \\
& \mathbf{x}_{q}=\mathbf{x}_{p}+z \mathbf{n}
\end{aligned}
$$


avec $\mathbf{u}_{\mathrm{p}}$ le vecteur déplacement de la surface moyenne, $\mathbf{n}^{0}$ et $\mathbf{n}$ sont les normales à la surface moyenne aux points $\mathrm{p}^{0}$ et $\mathrm{p}$. En utilisant les équations (Barlet et al., 1996) et (Batoz et al., 1998) nous calculons le tenseur de gradient de déformation $[\mathrm{F}]^{-1}$ ensuite le tenseur de Cauchy Green gauche $[\mathrm{B}]^{-1}=[\mathrm{F}]^{-\mathrm{T}}[\mathrm{F}]^{-1}$. Les deux élongations principales sont les valeurs propres du tenseur $[\mathrm{B}]^{-1}$.

Pour le calcul de contraintes, le critère de Hill 1948 (Lemaître et al., 1996) avec l'hypothèse d'anisotropie transverse est adopté :

$$
\phi=\frac{1}{2} \boldsymbol{\sigma}^{\boldsymbol{T}} \boldsymbol{P} \boldsymbol{\sigma}-\bar{\sigma}^{2}=0
$$

où $\bar{\sigma}(\bar{\varepsilon})$ est la contrainte limite élastique actualisée. $\mathbf{P}$ est la matrice d'anisotropie transverse définie par les coefficients de Lankford.

L'hypothèse du chargement proportionnel (Lemaître et al., 1996) permet l'intégration analytique des déformations de l'état initial à l'état final, d'où une loi constitutive totale :

$$
\left\{\begin{array}{l}
\sigma_{x x} \\
\sigma_{y y} \\
\sigma_{x y}
\end{array}\right\}=\frac{2}{3} \frac{\bar{\sigma}}{\bar{\varepsilon}}\left[\begin{array}{ccc}
F G & F & 0 \\
F & F H & 0 \\
0 & 0 & F K
\end{array}\right]\left\{\begin{array}{l}
\varepsilon_{x x} \\
\varepsilon_{y y} \\
\varepsilon_{x y}
\end{array}\right\}
$$

avec :

$$
2 \mathrm{~F}=2 \mathrm{G}=\frac{1}{\sigma_{\mathrm{y} 3}^{2}} ; \quad 2 \mathrm{H}=\frac{2}{\sigma_{\mathrm{y}}^{2}}-\frac{1}{\sigma_{\mathrm{y} 3}^{2}} ; \sigma_{\mathrm{y} 1}=\sigma_{\mathrm{y} 2}=\sigma_{\mathrm{y}} ; \quad \mathrm{K}=\frac{\sigma_{\mathrm{y}}}{\sigma_{\mathrm{y} 3}}
$$

La surface finale de l'embouti est discrétisée en éléments de coque de type DKT12 (Batoz et al., 1992). L'action de serre flan et de la matrice est remplacée par un vecteur de force

$$
\mathbf{f}=-2 \mu q_{n} \mathbf{t}
$$

avec $\mu$ le coefficient de frottement, f la force tangentielle de frottement par unité de surface, $\mathrm{q}_{\mathrm{n}}$ est la pression normale de serre-flan agissant sur les deux surfaces de la tôle, $\mathbf{t}$ est un vecteur unitaire défini par la direction de glissement de la tôle sous l'action du poinçon. L'action du poinçon est supposée être une pression nodale donnée par :

$$
\mathbf{P}=P \mathbf{n}^{\mathbf{f}} \text { avec } \quad \mathbf{n}^{\mathbf{f}}=\frac{1}{\sqrt{1+\mu^{2}}}(\mathbf{n}-\mu \mathbf{t})
$$




\subsection{Amélioration des contraintes de l'approche inverse}

\subsubsection{Idée de base}

Plusieurs travaux (Guo et al., 1990 ; Batoz et al., 1998 ; Ben-Elechi, 2004) ont montré que l'approche inverse permet une bonne estimation des déformations, mais l'estimation des contraintes reste entachée d'erreur (en particulier dans la région de brin libre entre la matrice et le poinçon) ce qui est dû principalement à l'ignorance de trajet de déformation. Dans ce qui suit nous présentons une technique géométrique pour tenir compte du trajet de déformation tout en gardant la propriété principale de l'approche inverse avec un seul pas de calcul.

\subsubsection{Technique d'approximation du trajet de déformation}

Soit une pièce emboutie dans ses deux configurations $C$ et $C^{0}$. Un point $p^{0}$ appartenant à un élément du flan initial est choisi. Le point $p^{0}$ occupera la position $p^{1}$ après emboutissage (figure 2). Afin de savoir si le point est passé par la ligne entrée de matrice, nous procédons de la façon suivante :

- nous projetons verticalement le vecteur de déplacement $\overrightarrow{p^{0} p^{1}}$ sur le maillage de la surface finale de l'embouti pour déterminer la courbe $p^{0^{\prime}} p^{1}$ qui appartient à la surface de l'embouti,

- pour savoir si le point $p^{0}$ est passé (ou non) par la ligne entrée de matrice, nous procédons par un calcul d'intersection entre la courbe $p^{0^{\prime}} p^{1}$ et la ligne entrée de matrice $c c$ '. Si l'intersection génère un point, nous concluons que le point a subi un cambrage et décambrage,

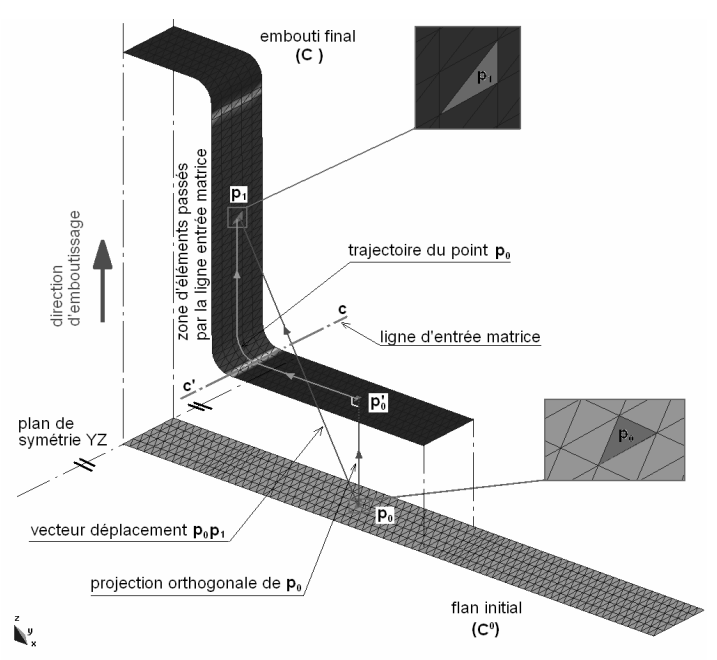

Figure 2. Approximation de la trajectoire de déformation d'un élément 
- tous les éléments identifiés sont ensuite modifiés par ajout de moments artificiels pour compenser les effets de cambrage et de décambrage non détectables par l'AI afin de tenir compte du trajet de déformation.

En général, il existe deux courbures au point d'intersection entre la courbe $p^{0} p^{1}$ et la ligne d'entrée matrice $c c^{\prime}$ (la courbure circonférentielle et la courbure radiale $\left.1 / R_{\text {die }}\right)$. D'après la théorie de flexion de poutres élastoplastiques, la composante de la déformation de poutre soumise à un moment de flexion est donnée par (figure 3) :

$$
\varepsilon_{\theta}=\ln \left(1+\frac{z}{r_{m}}\right)
$$

avec $r_{m}$ le rayon à la surface moyenne.

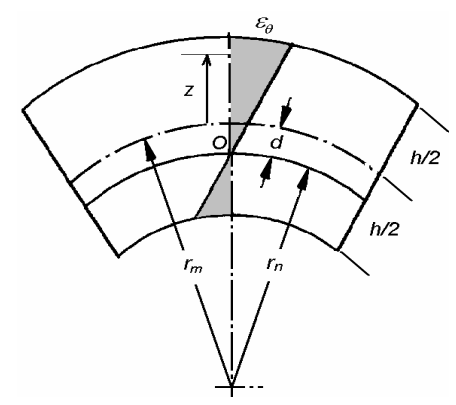

Figure 3. Pliage d'une poutre

La contrainte circonférentielle correspondante peut être calculée par :

$$
\begin{cases}\sigma_{\theta}=E \varepsilon_{\theta} & \rightarrow \text { si } \bar{\varepsilon} \leq \varepsilon_{y} \\ \sigma_{\theta}=\operatorname{sign}\left(\varepsilon_{\theta}\right) A K\left|\varepsilon_{\theta}\right|^{n} \rightarrow & \text { si } \bar{\varepsilon}>\varepsilon_{y}\end{cases}
$$

avec $E$ le module d'Young, $K$ et $n$ les paramètres d'écrouissage, $\varepsilon_{y}$ la limite élastique. $\bar{\varepsilon}$ la déformation équivalente et $A$ un coefficient d'anisotropie (Lemaître et al., 1996). Le moment de flexion peut être exprimé comme la somme d'une partie élastique et d'une partie plastique.

$M=M^{e}+M^{p}=\left(\int_{-z_{e}}^{z_{e}} z E \varepsilon_{\theta} d z\right)+\left(-\int_{-h / 2}^{-z_{e}} z A K\left|\varepsilon_{\theta}^{n}\right| d z+\int_{z_{e}}^{h / 2} z A K \varepsilon_{\theta}^{n} d z\right)$

où $z_{e}$ est la limite entre les régions élastique et plastique. Le moment élastique peut être approché numériquement en utilisant un schéma d'intégration de type Lobatto : 


$$
M^{e}=\sum_{i=1}^{n p l}\left(\omega_{i} z_{e}^{2} E \zeta_{i} \ln \left(1+\frac{z_{e} \zeta_{i}}{r_{m}}\right)\right)
$$

avec $n p l$ le nombre de points d'intégration et $\omega_{i}$ les poids correspondants. De même pour le calcul du moment plastique on utilise un schéma d'intégration de type Lobatto, on obtient ainsi :

$$
\begin{aligned}
& M^{p}=A K \sum_{i=1}^{n p l} \omega_{i}\left(\frac{z_{e}}{2}\left(1-\zeta_{i}\right)+\frac{h}{4}\left(1+\zeta_{i}\right)\right)\left(\frac{h}{4}-\frac{z_{e}}{2}\right)\left(\ln \left(1+\frac{\left(0.5 z_{e}\left(1-\zeta_{i}\right)+0.25 h\left(1+\zeta_{i}\right)\right)}{r_{m}}\right)\right)^{\mathrm{n}} \\
& +A K \sum_{i=1}^{n p l} \omega_{i}\left(\frac{z_{e}}{2}\left(1+\zeta_{i}\right)+\frac{h}{4}\left(1-\zeta_{i}\right)\right)\left(\frac{h}{4}-\frac{z_{e}}{2}\right)\left(-\ln \left(1+\frac{\left(-0.5 z_{e}\left(1+\zeta_{i}\right)-0.25 h\left(1-\zeta_{i}\right)\right)}{r_{m}}\right)\right)^{\mathrm{n}}
\end{aligned}
$$

Les moments de cambrage sont introduits pour chaque élément dans la zone de brin libre pour tenir compte des effets de cambrage et décambrage.

\section{Traitement du problème d'optimisation}

Dans tout problème d'optimisation, le but est de minimiser une fonction (Vanderplaats 1984) :

$$
\min f(\mathbf{x}), \mathbf{x} \in R^{n}
$$

vérifiant les inégalités suivantes :

$$
g_{j}(\mathbf{x}) \leq 0, j=1, \ldots, m
$$

et

$$
L_{i} \leq x_{i} \leq U_{i}, i=1, \ldots, n
$$

avec $f$ la fonction coût, les variables de conception, $g_{j}$ les limitations non linéaires. Pour les variables de conception le domaine de définition est délimité par des bornes inférieure et supérieure $\left(L_{i}, U_{i}\right)$. La méthode de surface de réponse MSR consiste à résoudre un problème où la fonction coût (et les limitations) est remplacée par des approximations $\tilde{f}$ (et $\tilde{g}_{j}$ ).

Le problème devient :

$$
\min \tilde{f}(\mathbf{x}), \mathbf{x} \in R^{n}
$$

avec 


$$
\tilde{g}_{j}(\mathbf{x}) \leq 0, j=1, \ldots, m
$$

Les approximations [16] et [17] sont obtenues en utilisant un échantillonnage de la fonction $f$. Le problème de distribution des points d'échantillonnage dans l'espace d'approximation est appelé plan d'expérience (PE). Le but est d'obtenir une meilleure approximation avec un nombre limité d'évaluations de $f$ et de $g_{j}$.

\section{Surface de réponse}

Les fonctions d'approximation utilisées dans la méthode de surface de réponse sont principalement linéaires ou de second ordre (Myers et al., 2002). Les coefficients d'approximation sont calculés par la méthode des moindres carrés. L'inconvénient est que ces modèles sont globaux sur une zone d'intérêt. L'idée appliquée par (Stander, 2001) est de faire la progression d'algorithme de minimisation en construisant de nouvelles surfaces de réponse centrées sur chaque minimum consécutif. Pendant la progression de la procédure, la zone d'intérêt se déplace et de nouvelles évaluations numériques sont réalisées à chaque itération. Cependant, la continuité entre les approximations n'est pas garantie et les informations qui sont obtenues aux itérations antérieures sont difficilement exploitables. Dans le travail présent, nous étudions la méthode de surface de réponse mobile. L'idée est d'utiliser des grilles non uniformes de points d'évaluations stockés durant les itérations successives. L'approximation étant locale, seuls les points qui sont proches de l'optimum courant sont pris en considération. Dans la suite, nous rappelons la formulation de la matrice d'approximation diffuse (Nayroles et al., 1992 ; Belytschko et al., 1994) et nous présentons notre modèle.

Connaissant les valeurs de la fonction coût pour un ensemble de points expérimentaux donnés par un plan d'expérience choisi, la fonction $\tilde{f}$ peut être définie par les fonctions de base $\mathbf{p}$ et des paramètres $\mathbf{a}$ :

$$
\tilde{f}(\mathbf{x})=\mathbf{p}^{T}(\mathbf{x}) \mathbf{a}(\mathbf{x})
$$

Les fonctions de base $\mathbf{p}$ sont choisies telles que :

$$
\left.\tilde{f}(\mathbf{x})=\left\langle\begin{array}{llllllll}
1 & x_{1} & x_{2} & \ldots & x_{n}
\end{array} x_{1} x_{2} \ldots x_{i} x_{i+1} \ldots x_{n-1} x_{n} ; x_{1}^{2} / 2 \ldots x_{n}^{2} / 2\right\rangle \begin{array}{l}
a_{0} \\
a_{1} \\
\vdots \\
a_{n-1} \\
a_{n}
\end{array}\right\}
$$

Les coefficients $a_{i}$ sont déterminés par la méthode des moindres carrés pondérée en minimisant l'écart $J(\mathbf{a})$ entre les points d'évaluation et les valeurs de la fonction objectif approchée. 


$$
J(\mathbf{a})=\sum_{i=1}^{N} w\left(\left\|\mathbf{x}_{i}-\mathbf{x}\right\|\right)\left(\mathbf{p}^{T}\left(\mathbf{x}_{i}-\mathbf{x}\right) \mathbf{a}-f\left(\mathbf{x}_{i}\right)\right)^{2}
$$

où $N$ est le nombre des points d'évaluation de la fonction coût et $\mathbf{x}_{i}$ les variables de la conception $w_{i}$ représentent les poids qui assurent la continuité et la localité de l'approximation.

La minimisation de la fonction objectif $J(\mathbf{a})$ donne :

$\mathbf{a}(\mathbf{x})=\mathbf{A}^{-1} \mathbf{B f}$

avec

$$
\begin{aligned}
& \mathbf{A}=\mathbf{P W} \mathbf{P}^{T} \\
& \mathbf{B}=\mathbf{P W} \\
& \mathbf{P}=\left[\begin{array}{lll}
\ldots & \mathbf{p}\left(\mathbf{x}_{i}-\mathbf{x}\right) & \ldots
\end{array}\right], \mathbf{W}=\left[\begin{array}{cccc}
w\left(\mathbf{x}_{1}-\mathbf{x}\right) & & & 0 \\
& w\left(\mathbf{x}_{2}-\mathbf{x}\right) & & \\
& & \ddots & \\
0 & & & w\left(\mathbf{x}_{n}-\mathbf{x}\right)
\end{array}\right]
\end{aligned}
$$

\section{Plan d'expériences}

La méthode de surface de réponse utilise un ensemble de points dans l'espace de conception pour déterminer un optimum approximatif. La progression se fait en se déplaçant ou en zoomant autour d'un point. Le passage d'une itération à une autre est obtenu en utilisant une méthode de descente appliquée à la surface de réponse. La figure 4 montre l'évolution possible d'une tête chercheuse à 4 points et à deux variables entre les itérations $i$ et $i+1$. A l'itération $i$, le point est le centre de la zone courante d'intérêt. La translation de la tête chercheuse pour la prochaine itération est donnée par une méthode de descente appliquée à la surface de réponse obtenue par les points noirs.

L'évolution de la taille de la tête chercheuse dépend de la nature de la solution et de l'exactitude de l'optimum courant. Sur la figure 4, la grille à l'itération i+1 est donnée par les points gris. Cependant, cette approche ne prend pas en compte les conceptions calculées à l'itération précédente, même lorsqu'elles appartiennent à la nouvelle zone d'intérêt, telle que le point noir en haut à droite de la figure 4 . Néanmoins, la nouvelle surface de réponse établie sans préserver la continuité avec la précédente peut mener à des oscillations et à la divergence. 


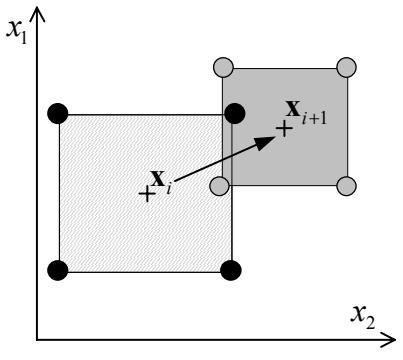

Figure 4. Evolution d'une tête chercheuse

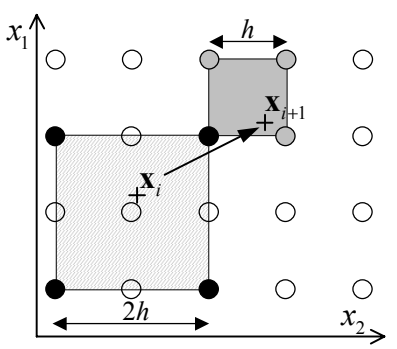

Figure 5. Points d'expérience virtuels

Sur la figure 5 les points creux montrent un ensemble discret de points dans l'espace de conception où les évaluations numériques sont autorisées, les points gris correspondent aux nouvelles évaluations de la fonction coût. Pour les points d'expérience virtuels, les évaluations sont conçues à ce stade mais pas encore exécutées. L'idée de base est qu'une conception virtuelle est évaluée seulement quand elle décrit la zone réelle d'intérêt.

Une approximation quadratique de la méthode des moindres carrés adaptable est obtenue lorsqu'on considère les coefficients $a_{i}$, donnés par [21]. Dans ce cas une conception optimale est obtenue explicitement par la résolution du système linéaire à nxn équations.

$$
\tilde{\mathbf{H}} \Delta \mathbf{x}=-\tilde{\nabla} f
$$

$\tilde{\mathbf{H}}$ et $\tilde{\nabla}$ sont respectivement le Hessian et le gradient de $f$ et $\Delta \mathbf{x}$ est le déplacement suivant.

Dans des cas localement non convexes un traitement spécial doit être mis en place. Le repositionnement spectral du Hessian, ou l'analyse d'arrête comme développés par (Myers et al., 2002) doivent être employés.

Pour une grille donnée de points, on décompose la matrice $\mathbf{B}$ en produit de deux matrices.

$$
\mathbf{B}=\mathbf{Q}(h) \mathbf{C}
$$

C dépend de la distribution des points sur la grille et $\mathbf{Q}(h)$ est une matrice diagonale qui dépend uniquement de la taille $h$ de la grille. Pour la base quadratique on a :

$$
\mathbf{Q}=\left[\begin{array}{ccc}
1 & 0 & 0 \\
0 & h \mathbf{I}_{2^{* 2}} & 0 \\
0 & 0 & h^{2} \mathbf{I}_{3^{*}}
\end{array}\right]
$$


En substituant [25] dans [21] on obtient :

$$
\mathbf{a}(\mathbf{x})=\mathbf{Q}^{-1} \mathbf{D f}_{n}
$$

où la matrice $\mathbf{D}$ est donnée par :

$$
\mathbf{D}=\left[\mathbf{C W C}^{T}\right]^{-1} \mathbf{C W}
$$

D est calculée seulement pour une grille de référence et l'évaluation de [27] est simplifiée du faite que la matrice $\mathbf{Q}$ est diagonale. $\Delta \mathbf{x}$ :

En introduisant [28] et [26] dans [27] ont obtient une expression implicite de

$$
\begin{aligned}
\Delta x_{1}= & \frac{r}{2 d}\left(\left(2 f_{1}+f_{2}+f_{3}-f_{4}-f_{5}\right)\left(f_{3}-f_{2}\right)+\left(f_{7}+f_{9}\right)\left(f_{1}+f_{2}\right)\right. \\
& \left.-\left(f_{6}+f_{8}\right)\left(f_{1}+f_{3}\right)-f_{7}\left(f_{5}+f_{9}\right)-f_{9}\left(f_{4}-f_{8}\right)+f_{4} f_{8}+f_{5} f_{6}\right) \\
\Delta x_{2}= & \frac{r}{2 d}\left(\left(2 f_{1}-f_{2}-f_{3}+f_{4}+f_{5}\right)\left(f_{5}-f_{4}\right)+\left(f_{7}-f_{9}\right)\left(f_{2}-f_{1}\right)\right. \\
& \left.+\left(f_{6}-f_{8}\right)\left(f_{3}-f_{1}\right)+f_{7}\left(f_{6}-f_{5}\right)+f_{9}\left(f_{4}-f_{8}\right)+f_{4} f_{8}-f_{5} f_{6}\right)
\end{aligned}
$$

avec $d=\left(f_{2}+f_{3}-f_{4}-f_{5}\right)^{2}-\left(f_{6}+f_{9}-2 f_{1}\right)\left(f_{7}+f_{8}-f_{1}\right)$

\section{Algorithme d'optimisation}

Le but du processus d'optimisation est de chercher le point optimal de la fonction objectif. L'algorithme d'optimisation est donné par :

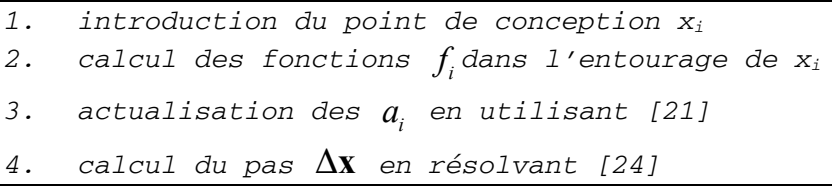

L'étape 2 consiste à rechercher les points d'évaluation sur les points déjà présents puis à calculer les points qui manquent.

\section{Applications numériques}

\subsection{Emboutissage de la bôte carrée de Numisheet'93}

Nous avons effectué notre analyse sur la boîte carrée proposée de manière détaillée comme benchmark à la conférence internationale de Numisheet (1993) 
(Makinouchi et al., 1993). Il s'agit de simuler l'emboutissage d'une boîte carrée de profondeur $25 \mathrm{~mm}$, à partir d'un flan carré de $150 \mathrm{~mm}$ x $150 \mathrm{~mm}$ (figure 6). Nous avons utilisé 8628 élément de DKT12 (figure 7) pour la simulation avec l'AI classique et l'AIA, et 4096 éléments quadrangulaires de coque S4R pour la simulation avec Abaqus ${ }^{\circledR}$ (statique implicite).
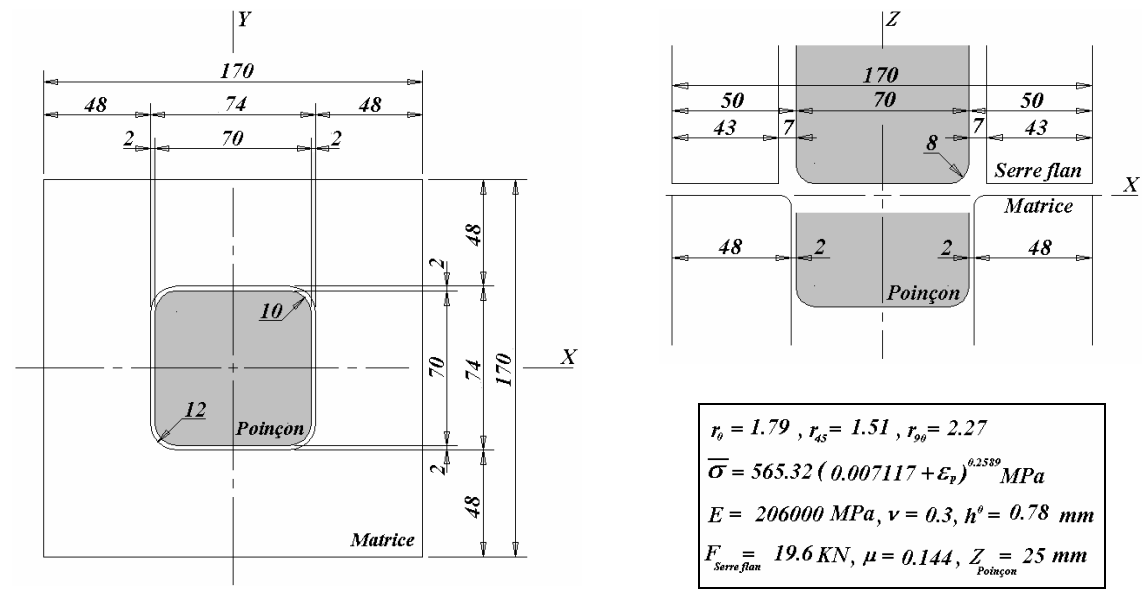

Figure 6. Description géométrique et mécanique de la boîte carrée

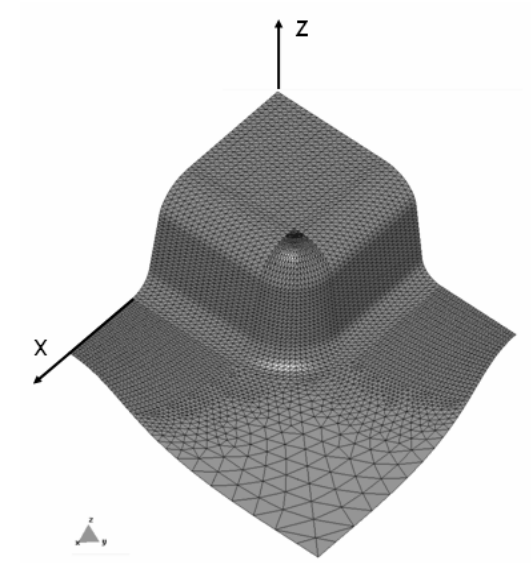

Figure 7. Maillage du quart de la boite carrée (8 626 éléments DKT12)

Pour comparer les résultats obtenus, nous avons tracé l'évolution de la contrainte maximale sur la fibre supérieure le long du plan de symétrie XZ (figure 8). Nous remarquons que, contrairement à l'AI classique, l'AIA estime bien la répartition des 
contraintes dans le brin libre de la tôle (région entre le poinçon et la matrice $40 \mathrm{~mm} \leq s \leq 60 \mathrm{~mm}$ ).

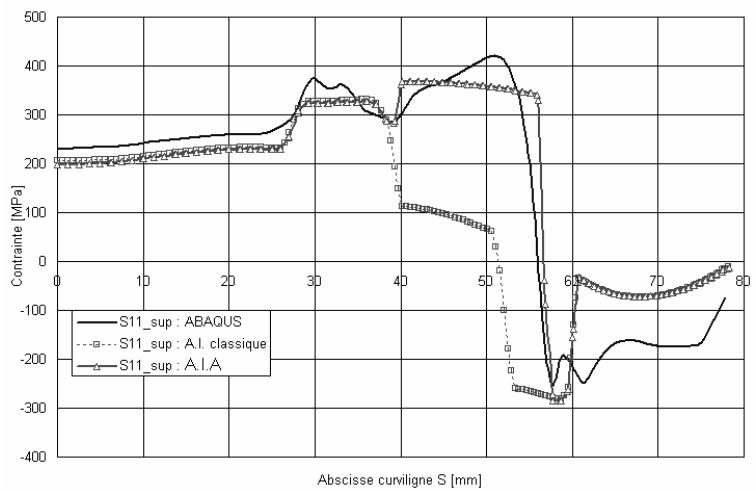

Figure 8. Distribution de la contrainte maximale sur la fibre supérieure

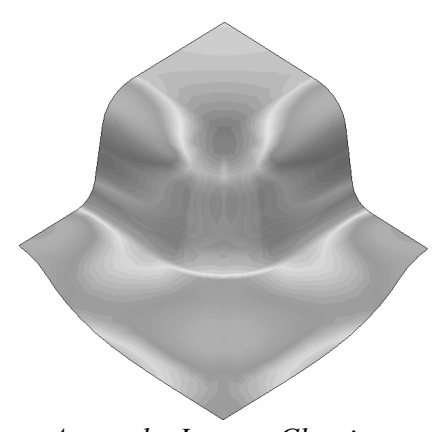

Approche Inverse Classique

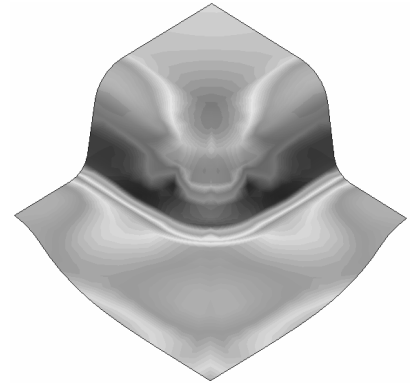

Approche Inverse Améliorée

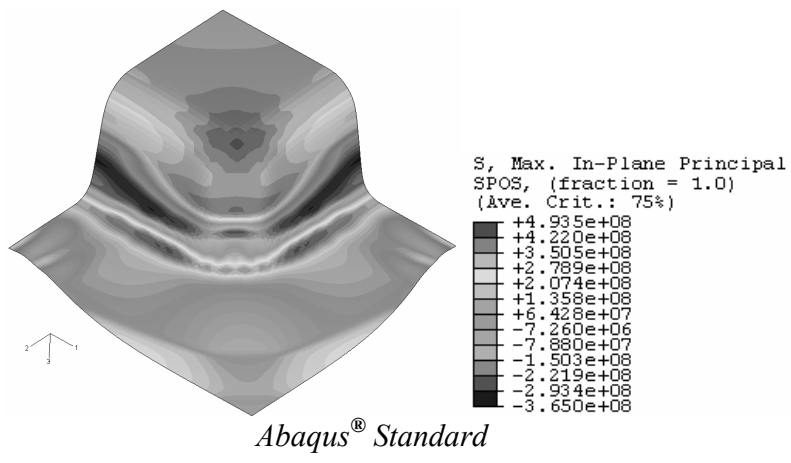

Figure 9. Distribution de la contrainte maximale sur la fibre supérieure 
La figure 9 montre la distribution en iso-couleurs de la contrainte maximale sur la fibre supérieure obtenue par l'AIC, L'AIA et Abaqus ${ }^{\circledR}$ standard. Nous remarquons que l'AIA détecte les éléments qui ont subi les effets de cambrage/decambrage et améliore l'estimation des contraintes dans la partie du brin libre (zone entre les rayons poinçon et le rayon matrice). Donc la prise en compte du trajet de déformation permet d'améliorer l'estimation des contraintes.

\subsection{Emboutissage de la tôle en forme de " $U$ "}

Cette application concerne l'emboutissage de test «U» (figure 10), qui a été proposé dans Numisheet'93 (Makinouchi et al., 1993). Après l'opération d'emboutissage et le retrait des outils, la forme en «U » n'est pas conservée. Notre but est de déterminer les paramètres d'ouverture de la tôle : $\theta_{1}, \theta_{2}$ et $\rho$ (voir figure 11).

La tôle rectangulaire a une largeur de $35 \mathrm{~mm}$ et une longueur de $350 \mathrm{~mm}$ en acier. La loi d'écrouissage est de type isotrope de Krupkowsky-Swift $\bar{\sigma}=K\left(\varepsilon_{0}+\bar{\varepsilon}^{p}\right)^{n}$. Les caractéristiques géométriques et matérielles de la tôle sont données dans le tableau 1 .

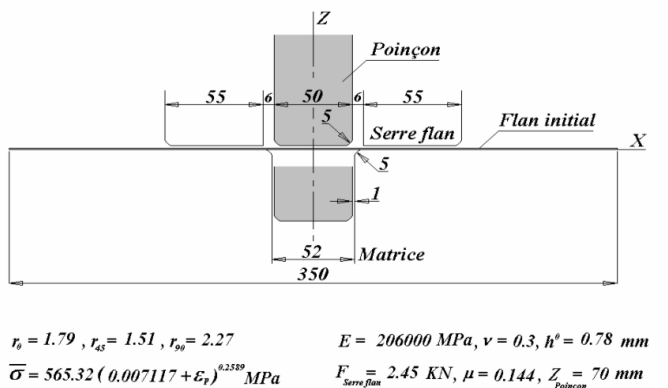

Figure 10. Description mécanique et géométrique

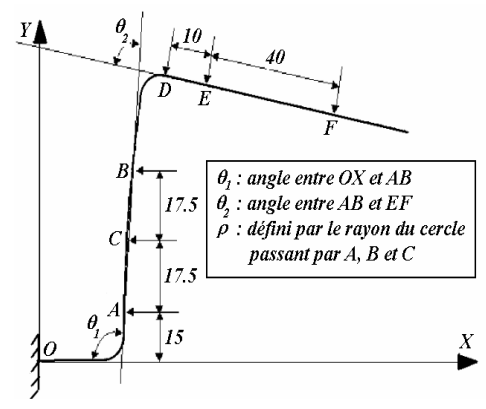

Figure 11. Retour élastique après le retrait des outils

Les figures 12 et 13 montrent les répartitions de la contrainte axiale sur la peau inférieure, obtenue par Abaqus ${ }^{\circledR}$ standard et l'approche inverse.

Ici on considère uniquement la non-linéarité géométrique. Nous avons utilisé dix pas de chargements avec une précision de $10^{-6}$ sur la norme euclidienne de déplacement. La figure 14 montre les formes initiale et finale de la tôle en « $U$ » (avant et après l'enlèvement des outils) obtenue en utilisant notre modèle. 


\begin{tabular}{lcccc}
\hline \multicolumn{1}{c}{ Propriété } & & Valeur & \\
module d'Young & $\mathrm{E}$ & 206 & $\mathrm{GPa}$ \\
Coefficient de Poisson & $v$ & 0.3 & \\
Epaisseur initiale & $h_{0}$ & 0.78 & $\mathrm{~mm}$ \\
Limite élastique initiale & $\bar{\sigma}_{e}$ & 157 & $\mathrm{MPa}$ \\
Coefficient d'écrouissage & $\mathrm{K}$ & 565.32 & $\mathrm{MPa}$ \\
Coefficient d'écrouissage & $\varepsilon_{0}$ & 0.007117 & \\
Coefficient d'écrouissage & $\mathrm{n}$ & 0.2589 & \\
Coefficient d'anisotropie & $r_{00}, r_{45}, r_{90}$ & $1.79,1.51,2.27$ & \\
densité & $\rho$ & 7800 & $\mathrm{Kg} / \mathrm{m}^{3}$ \\
Coefficient de frottement (tôle/outil) & $\mu$ & 0.144 & \\
Force de serrage & F & 2.45 & $\mathrm{kN}$ \\
\hline
\end{tabular}

Tableau 1. Caractéristiques matérielles et géométriques

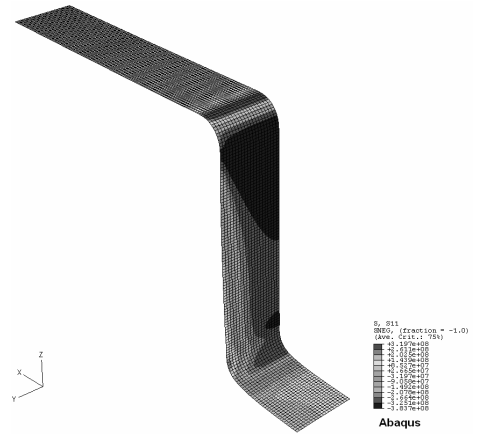

Figure 12. Distribution de la contrainte axiale - peau inférieure $\left(\right.$ Abaqus $\left.^{\circledR}\right)$

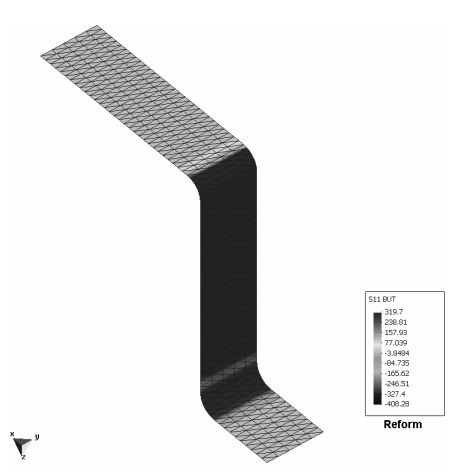

Figure 13. Distribution de la contrainte axiale - peau inférieure (approche inverse) 


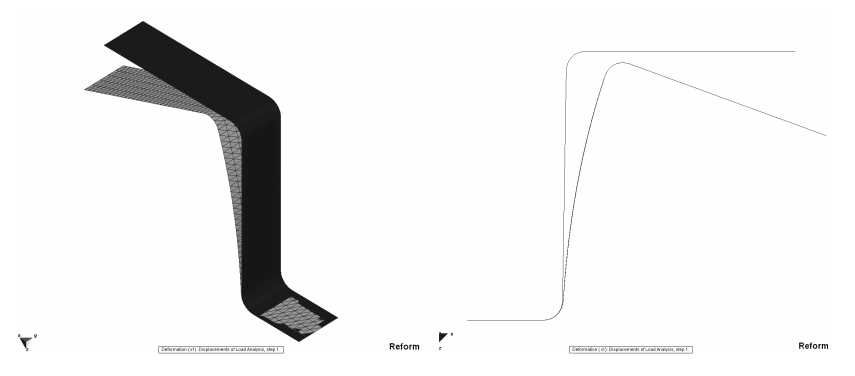

Figure 14. Test «U $U$ » avant et après retrait des outils

\begin{tabular}{|c|c|c|c|c|c|}
\hline Méthode & Nb d'éléments & $\theta_{1}$ & $\theta_{2}$ & $\rho$ & Temps CPU \\
\hline $\begin{array}{l}\text { Dynamique explicite } \\
\left(\text { Stampack }^{\circledR}\right)\end{array}$ & BST (4000) & 97.98 & 80.02 & 335.01 & $1 \mathrm{~h} 18 \mathrm{~m} \mathrm{49s}$ \\
\hline $\begin{array}{l}\text { Statique implicite } \\
\text { (Abaqus }^{\circledR} \text { ) }\end{array}$ & S4R (4640) & 97.88 & 80.98 & 239.09 & $140 \mathrm{~h} 15 \mathrm{~m} 02 \mathrm{~s}$ \\
\hline $\begin{array}{l}\text { GATI } \\
\text { (A.P.I. avec } 16 \text { pas) }\end{array}$ & $\begin{array}{l}\text { DKTRF } \\
(9280)\end{array}$ & 104 & 81 & 154 & $1 \mathrm{~h} 20 \mathrm{~m} \mathrm{22s}$ \\
\hline $\begin{array}{l}\text { Approche inverse en } \\
1 \text { pas (REFORM) }\end{array}$ & $\begin{array}{l}\text { DKT12 } \\
(800)\end{array}$ & 99.94 & 80.08 & 233.62 & $0 \mathrm{~h} 00 \mathrm{~m} 05 \mathrm{~s}$ \\
\hline \multirow{2}{*}{ Numisheet'93 } & Simulation & 99 & 82 & 240 & \\
\hline & Expérience & 99.2 & 82.1 & ---- & \\
\hline
\end{tabular}

Tableau 2. Résultats de retour élastique. Simulations par différents codes

Nous présentons sur le tableau 2 les moyennes des résultats expérimentaux et de simulations de Numisheet'93. Si on s'intéresse au temps CPU, nous remarquons d'abord en comparant les deux codes utilisant des approches incrémentales qui nous servent de référence, que le calcul sur Stampack ${ }^{\circledR}$ est environ 107 fois plus rapide que le calcul sur Abaqus ${ }^{\circledR}$ standard. Cette différence s'explique par le fait qu'en statique implicite, à chaque pas, on doit vérifier l'équilibre entre les forces internes et externes, donc la taille du pas doit être très petite pour assurer la convergence, chose qui n'est pas vérifiée en dynamique explicite. Les algorithmes de gestion du contact ne sont pas non plus les mêmes.

Nous remarquons, par ailleurs, que le temps de calcul de l'AI est très faible par rapport à toutes les autres méthodes y compris celle de (Gati et al., 2003) employant l'approche pseudo-inverse avec 16 pas (Guo et al., 2001). Concernant le retour 
élastique nous remarquons que nos résultats sont très proches de ceux obtenus avec Abaqus $^{\circledR}$ ainsi que ceux de Numisheet'93. Les résultats de Stampack ${ }^{\circledR}$ et de l'approche pseudo-inverse (Gati et al., 2003) restent relativement loins de la solution de référence et ce, même avec un maillage assez fin.

\section{Optimisation des rayons d'outils}

L'objectif est de déterminer les rayons optimaux des outils pour minimiser l'ouverture $" \mathrm{~d}_{\mathrm{i}}$ " (figure 15). On considère deux rayons, le rayon poinçon $R_{p}$ et le rayon matrice $R_{d}$. On choisit $\mathrm{R}_{\mathrm{p}}=\mathrm{R}_{\mathrm{d}}=5 \mathrm{~mm}$ comme valeurs initiales pour l'optimisation. La fonction objectif est donnée par :

$$
J=\sum_{i=1}^{n n t} d_{i}^{2}
$$

où $d_{i}$ représente le déplacement total de chaque point matériel après retrait des outils (détente de la pièce).

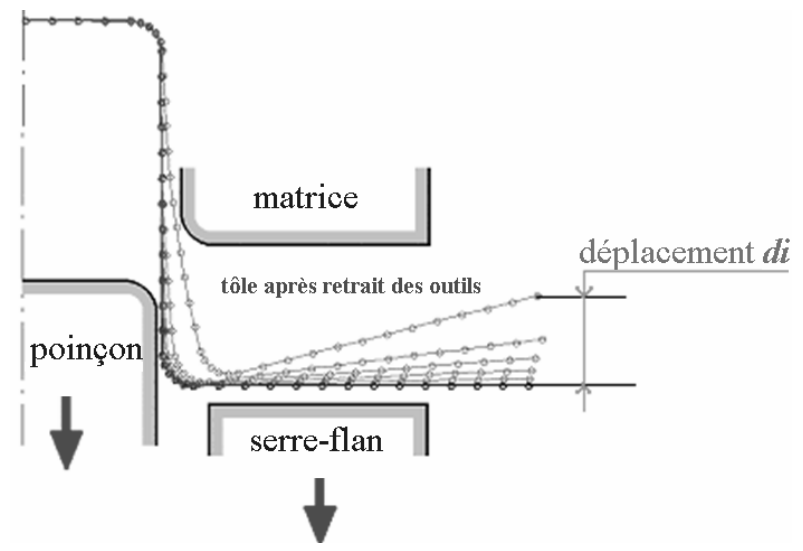

Figure 15. Test « $U$ » avant et après le retrait des outils

Le problème d'optimisation est résolu avec la procédure d'optimisation globale. Une grille initiale de $6 \times 6$ points équidistants est utilisée pour évaluer les vraies fonctions objectifs avec les limitations : $R_{\min }=2 \mathrm{~mm}$ et $R_{\max }=23 \mathrm{~mm}$. Les figures 16 et 17 montrent respectivement, la « vraie » fonction objectif et le modèle de surface de réponse obtenu par l'approximation diffuse. Le minimum obtenu correspond à $\left(R_{p}=4.130, R_{d}=21.401\right)$. 


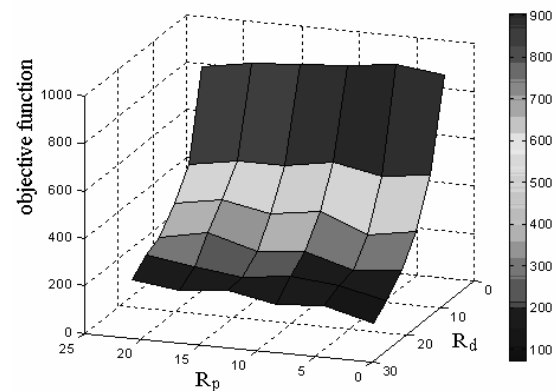

Figure 16. " Vraie » fonction objectif

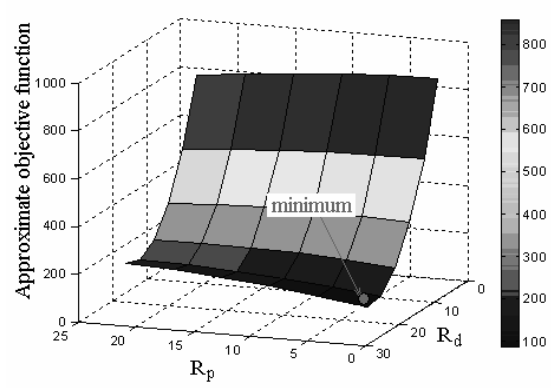

Figure 17. Modèle de surface de réponse

Sur la figure 18 nous avons présenté les résultats du retour élastique du test d'ouverture de la tôle en $U$ avant et après optimisation. Nous remarquons que la distance maximale de l'ouverture de la tôle est passée de $d_{i}=22.9 \mathrm{~mm}$ à $d_{i}=8.6 \mathrm{~mm}$.

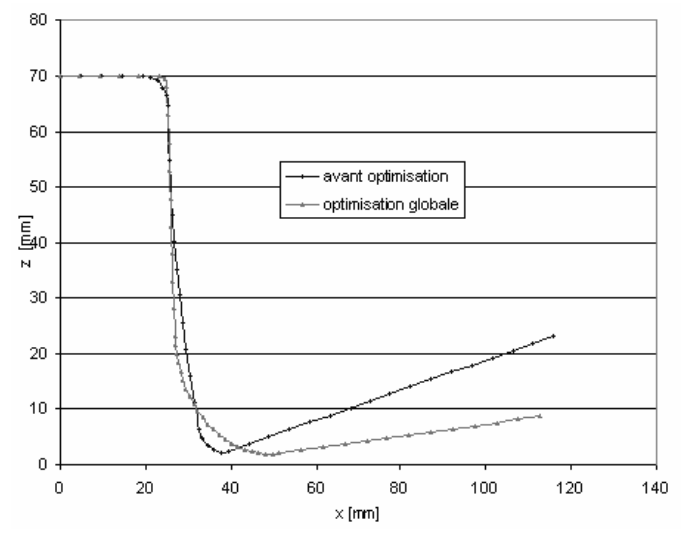

Figure 18. Retour élastique avant et après optimisation

\subsection{Emboutissage du godet axisymétrique}

Nous avons étudié le test proposé par le Laboratoire Ford (USA) (Demeri et al., 2000). Les caractéristiques géométriques et mécaniques sont données sur la figure 19. Le modèle de maillage utilisé pour modéliser le quart du godet est de 3150 éléments de coque DKT12 (figure 20). 

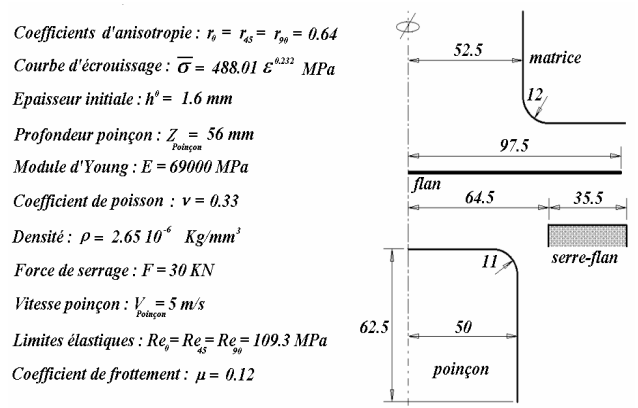

Figure 19. Description mécaniques et géométrique du test axisymétrique

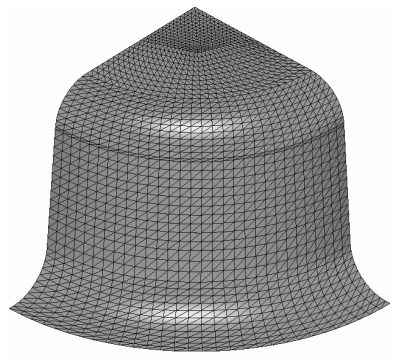

Figure 20. Maillage du quart $d u$ godet (3150 DKT12)

Ce test est un exemple complet du fait qu'il y a une opération d'emboutissage suivie par deux opérations de découpage (figure 21).

La figure 22 montre la distribution des contraintes circonférentielles sur la fibre supérieure. Nous pouvons constater qu'il y a une nette différence entre les résultats obtenus par notre AI améliorée et l'AI classique surtout dans la zone du brin libre (la zone entre le rayon poinçon et le rayon matrice). Nous remarquons également que nos résultats sont en bonne concordance avec ceux obtenus par Abaqus ${ }^{\circledR}$ Standard qui utilise une approche statique implicite. Ceci prouve que les corrections apportées à l'AI permettent d'améliorer l'estimation des contraintes.

A la fin de la simulation d'emboutissage nous avons effectué une découpe horizontale du godet cylindrique, suivant les deux lignes $C_{1}$ et $C_{2}$, suivie d'une deuxième découpe verticale suivant $\mathrm{C}_{3}$ (figure $21 \mathrm{~b}$ ). Les efforts internes de membrane et de flexion obtenus en fin d'emboutissage sont utilisés pour faire la décharge.

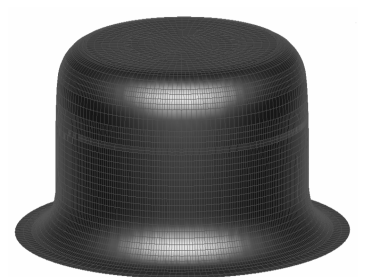

a)

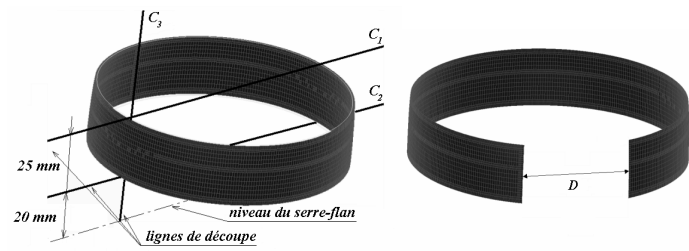

b)

Figure 21. Forme de l'embouti et opérations de découpe

La simulation du retour élastique est faite en utilisant l'élément de coque DKT12 avec une approche incrémentale basée sur la formulation lagrangienne actualisée. Dix pas de chargement sont pris avec une précision de $10^{-6}$ sur la norme euclidienne des déplacements. La figure 23 montre les formes de la demi-couronne (avant et 
après retrait des outils) obtenues en utilisant notre modèle d'AI améliorée. Les principaux résultats du retour élastique sont résumés sur le tableau 3.

\begin{tabular}{|l|c|c|c|c|}
\hline \multicolumn{2}{|c|}{ Méthode } & Nb. d'éléments & $\begin{array}{c}\text { Dmoy } \\
\text { [mm] }\end{array}$ & Temps CPU \\
\hline $\begin{array}{l}\text { Dynamique explicite } \\
\left(\text { Stampack }^{\circledR}\right)\end{array}$ & FLAI 15 pas & $\begin{array}{c}7200 \mathrm{BST} \\
(1 \text { godet })\end{array}$ & 45.6 & $2 \mathrm{~h} 33 \mathrm{mn} 53 \mathrm{sec}$. \\
\hline $\begin{array}{l}\text { Statique implicite } \\
\left(\text { Abaqus }^{\circledR}\right)\end{array}$ & FLAI 20 pas & $\begin{array}{c}6600 \mathrm{~S} 3 \mathrm{R} \\
(1 \text { godet })\end{array}$ & 48.6 & $7 \mathrm{~h} 09 \mathrm{mn} 48 \mathrm{sec}$. \\
\hline AIA (notre travail) & FLAI 20 pas & $\begin{array}{c}\text { DKT12 } \\
(1 / 4 \text { du godet })\end{array}$ & 45.6 & $18 \mathrm{sec}$. \\
\hline Expérience & & & 50 & \\
\hline
\end{tabular}

Tableau 3. Principaux résultats du retour élastique

Tout d'abord nous pouvons remarquer que les résultats obtenus par Abaqus ${ }^{\circledR}$ sont très proches de l'expérience mais avec un temps de calcul très élevé. Remarquons toutefois que les résultats obtenus par l'approche inverse améliorée sont en bonne concordance avec ceux de l'expérience, ils sont également proches de ceux obtenus avec Stampack ${ }^{\circledR}$ mais avec un temps CPU beaucoup plus faible.

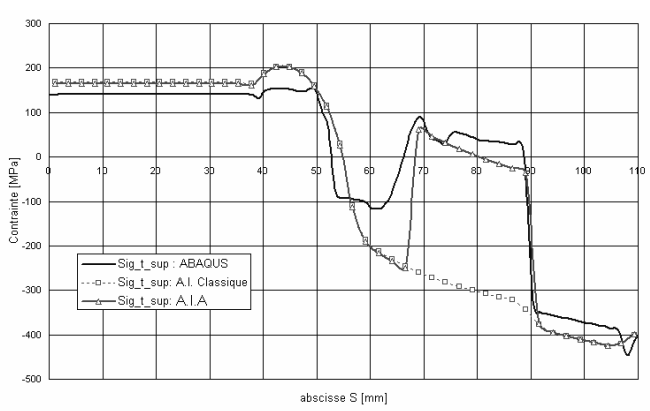

Figure 22. Distribution des contraintes circonférentielles sur la fibre supérieure

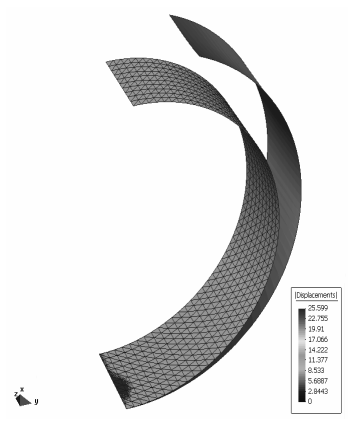

Figure 23. Configurations avant et après retour élastique

\section{Optimisation de la géométrie des outils}

L'objectif ici est de trouver le rayon de matrice optimal qui permet de minimiser l'ouverture de la couronne cylindrique après le retour élastique. Nous avons choisi comme fonction objective la norme $\mathrm{L}_{2}$ des déplacements de tous les nœuds. Nous avons choisi une valeur initiale du rayon matrice égale à $12 \mathrm{~mm}$. Nous avons utilisé une tête chercheuse à trois points et une taille de $1 \mathrm{~mm}$ avec une tolérance de $10^{-3}$ sur la valeur de la fonction coût. 


$$
J=\sqrt{\sum_{i=1}^{n n t} d_{i}^{2}}=\sqrt{\sum_{i=1}^{n n t}\left(U_{X i}^{2}+U_{Y i}^{2}+U_{Z i}^{2}\right)}
$$

Les figures 24 et 25 montrent la convergence des résultats d'optimisation en utilisant la méthode des moindres carrés mobiles. Seules trois itérations ont été nécessaires pour converger vers le minimum correspondant à un rayon de matrice maximal.

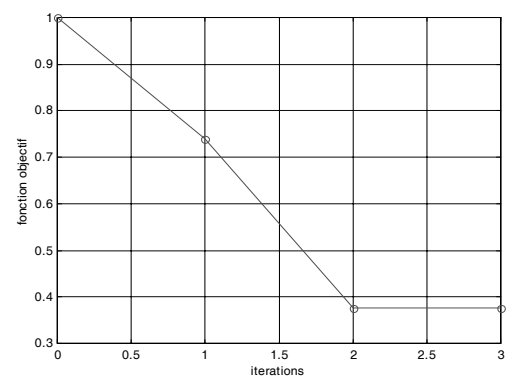

Figure 24. Convergence de la fonction coût

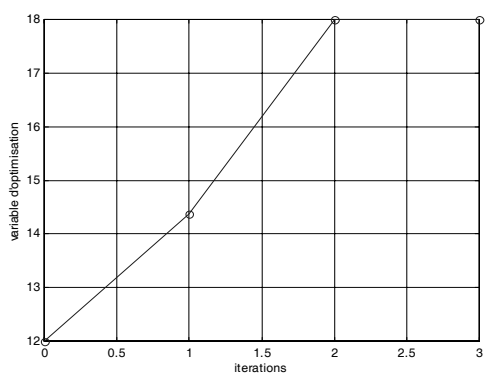

Figure 25. Convergence de la variable

Les figures 24 et 25 montrent l'ouverture de la couronne avant et après optimisation. Nous pouvons constater que la distance moyenne d'ouverture passe de 45,6 mm (avant optimisation) à 18,2 mm (après optimisation).

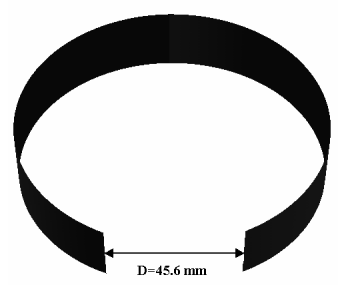

Figure 26. Ouverture de la couronne avant optimisation

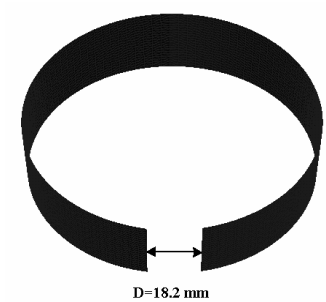

Figure 27. Ouverture de la couronne après optimisation

\section{Conclusion}

Dans l'AIA, les déformations et les contraintes dues aux effets de cambrage/décambrage sont calculées analytiquement en prenant en compte le trajet 
de déformation des éléments. La validation des résultats de l'approche inverse améliorée est faite en utilisant le logiciel Abaqus ${ }^{\circledR}$ Standard. Les efforts internes améliorés et la forme finale de la pièce emboutie sont utilisés pour le calcul du retour élastique en adoptant une formulation lagrangienne actualisée et en utilisant l'élément de coque DKT12. Les tests d'emboutissage, de la tôle en «U » et du godet axisymétrique sont étudiés et de bons résultats de la simulation du retour élastique sont obtenus avec un temps de calcul extrêmement faible. Une méthode de surface de réponse basée sur l'approximation diffuse et la technique de la tête chercheuse a été utilisée pour minimiser le retour élastique après retrait des outils. Les variables d'optimisation sont les rayons outils (poinçon, matrice). Les résultats obtenus montrent que le couplage de l'AIA avec l'algorithme d'optimisation par surface de réponse basée sur l'approximation diffuse permet une minimisation rapide et efficace du retour élastique.

\section{Bibliographie}

Barlet O., Batoz J.L., Guo Y.Q., Mercier F., Naceur H., Knopf-Lenoir C., "The inverse approach and mathematical programming techniques for optimum design of sheet forming parts", ASME, (3), 1996, p. 227-232.

Batoz J.L., Guo Y.Q., Mercier F., "The Inverse Approach with simple triangular shell elements for large strain predictions of sheet metal forming parts", Engineering Computations, vol. 15, n 6-7, 1998, p. 864-892.

Batoz J.L, Dhatt G., "Modélisation des structures par éléments finis”, vol. 3, Coques, Hermès, Paris, 1992.

Belytschko T., Lu Y.Y., Gu L., "Element-Free Galerkin Methods", International Journal for Numerical Methods in Engineering, 37, 1994, p. 229-256.

Ben Elechi S., Analyse et Conception Rapides de Paramètres du Procédé d'Emboutissage pour le Contrôle du Retour élastique, Thèse de doctorat, Université de Technologie de Compiègne, décembre 2004.

Bui Q., Papeleux L., Penthot J., "Efficient Springback Prediction based on Enhanced Assumed Strain Elements", $7^{\text {th }}$ International Conference on Computational Plasticity, April 7-10, 2003, Barcelona, Spain.

Delamézière A., Naceur H., Breitkopf P., Knopf-Lenoir C., Batoz J.L., Villon P., "Feasibility in deep drawing: optimization of material properties using response surface", Mécanique \& Industries, vol. $3, \mathrm{n}^{\circ} 2,2002$, p. 93-98.

Demeri M.Y., Lou M., Saran M.J., "A benchmark test for springback in sheet metal forming”, International Body Engineering Conference \& Exibition (IBEC), Cobo Center, Detroi, Michigan, Etat-Unis, October 3-5, 2000.

Gati W., Guo Y.Q., Naceur H., Batoz J.L., « Approche Pseudo Inverse pour estimation des contraintes dans les pièces embouties axisymétriques », Revue européenne des éléments finis, décembre 2003.

Gelin J.C., Picart P., "4 ${ }^{\text {th }}$ Int. Conf. Numerical Simulation of 3D Sheet Metal Forming processes”, Numisheet'99, Besançon, France, 1999. 
Guo Y. Q., Gati W., Naceur H., Batoz J. L., “An efficient DKT rotation free shell element for springback simulation in sheet metal forming", Computers \& Structures, vol. 80, $\mathrm{n}^{\circ} 27$ 30, November 2002, p. 2299-2312.

Guo Y.Q., Batoz J.L., Detraux J.M., Duroux P., "Finite element procedures for strain estimations of sheet metal forming parts", IJNME, (39), 1990, p. 1385-1401.

Guo Y.Q., Batoz J.L., Naceur H., Bouabdallah S., Mercier F., Barlet O., "Recent developments on the Analysis and Optimum Design of Sheet Metal Forming Parts using a Simplified Inverse Approach", Computers \& Structures, vol. 78, n 1-3, November 2000, p. 133-148.

Guo Y.Q., Gati W., Naceur H., Batoz J.L., "Stress evaluation in sheet forming modeling by inverse approach for springback simulation", EUROMAT 2001, 7th European Conf. on Advanced Materials and Processes, 10-14 june 2001, Rimini, Italy.

Guo Y.Q., Gati W., Naceur H., Batoz J.L., "Springback evaluation after forming simulation using the inverse approach and incremental approach", NUMIFORM 2001, 7th Int. Conf. on Num. Meth. In Industrial Forming Processes, June 18-21, 2001, Toyohashi, Japan.

Guo Y.Q., Naceur H., Debray K., Bogard F., "Initial solution estimation to speed up inverse approach in stamping modeling", International Journal for Computer-Aided Engineering, vol. $20, \mathrm{n}^{\circ} 7,2003$, p. 810-834.

Hibbit, Karlson, Sorensen, Abaqus ${ }^{\circledR} /$ Standard User's manual - version 6.3, Edition HKS, Pawtucket, RI, USA, 2003.

Ken-Ichiro M., "Simulation of material processing: theory, methods and applications", Numiform'2001, 7th Int. Conf. on Numerical Methods in Industrial Forming Processes, Toyohashi, Japan, 2001.

Kleiber M., Knabel J., Rojek J., "Reliability Assessment in Metal Forming Operations", $5^{\text {th }}$ World Cong. on Comp. Mech., editor Mang, H.A., Rammerstorfer F.G., Eberhardsteiner J., July 7-12, 2002, Austria.

Kurtaran H., Eskandarian A., Marzougui D., Bedewi N. E., "Crashworthiness design optimization using successive response surface approximations", Computational Mechanics, 29, 2002, p. 409-421.

Lancaster P., Salkauskas K., An Introduction: Curve and Surface Fitting, Academic Press Inc., 1986.

Lemaitre J., Chaboche J.L., Mécanique des matériaux solides, Dunod, Paris, 1996.

Lewis R.M., Torczon V., "Pattern Search Methods For Linearly Constrained Minimization", Siam J. Optim., vol. 10, n³, 2002, p. 917-941.

Makinouchi A., Nakamachi E., Oñate E., Wagoner R., Proceedings of the International Conference NUMISHEET'93, Riken, Tokyo, 1993.

Myers R.H., Montgomery D.C., Response Surface Methodology Process and Product Optimization using Designed Experiments, John Wiley and Sons, Inc., New York, USA, $2^{\text {nd }}$ ed., 2002.

Naceur H., Guo Y.Q., Batoz J.L., Bouabdallah S., Knopf-Lenoir C., "Design of process parameters in deep drawing of thin sheets using the simplified Inverse Approach", Numisheet'99, (1), France, 1999, p. 517-522. 
372 REMN - 17/2008. Optimisation des procédés de mise en forme

Naceur H., Guo Y.Q., Gati W., "New Enhancements in the inverse approach for the fast modeling of autobody stamping process", International Journal of Computational Enginering Science, vol. 3, n 4, 2002, p. 355-384.

Nayroles B., Touzot G., Villon P., "Generalizing the Finite Element Method: Diffuse Approximation and Diffuse Elements”, Computational Mechanics, 10, 1992, p. 307-318.

Quantech ATZ, Stampack ${ }^{\circledR}$ user guide - version 5.3, Edificio Nexus, Gran Capitán, 2-4, Barcelona, Spain, 2003.

Rojek J., Oñate E., "Sheet Springback Analysis Using a Simple Shell Triangle with Translational Degrees of Freedom Only", International Journal of Formin Processes, vol. 1, n 3, 1998, p. 275-296.

Roux W.J., Stander N., Haftka R.T., "Response surface approximations for structural optimization”, Int. J. Numer. Meth. Engng., 42, 1998, p. 517-534.

Stander N., "The successive response surface method applied to sheet-metal forming", Proceedings, First MIT Conference on Computational Fluid and Solid Mechanics, June 12-15 2001, p. 481-485

Stander N., Burger M., Zhu X., Maker B., “An Optimization Procedure For Springback Compensation Using LS-OPT", $7^{\text {th }}$ International LS-DYNA Users Conference, May 1921, Dearborn, Michigan, 2002, p. 15/1-10.

Vanderplaats G., Numerical Optimization Techniques for Engineering Design, McGraw Hill, 1984.

Yuen W.Y.D., "A generalized solution for the prediction of springback in laminated strip", Journal of Material Processing Tecnology, 61, 1996, p. 254-264.

Zhang T., Rahman S., Choi K.K., "A Hybrid Method using Response Surface and Pattern Search for Derivative-Free Design Optimization", $5^{\text {th }}$ World Cong. on Compt. Mech., editor Mang, H.A., Rammerstorfer F.G., Eberhardsteiner J., July 7-12, 2002, Austria. 